\title{
Regulating energy stores
}

Whether interleukin-4 (IL-4)driven $T$ helper $2\left(\mathrm{~T}_{\mathrm{H}} 2\right)$-type immune responses regulate nutrient metabolism and insulin sensitivity is unclear. In this study, Chawla and colleagues show that IL-4, through activation of signal transducer and activator of transcription 6 (STAT6), enhances the anabolic actions of insulin to promote storage of glucose. This is mediated by inhibition of catabolic metabolism in the liver and attenuation of inflammation in white adipose tissue.

IL-4 was shown to induce STAT6 activation in the liver, but not in skeletal muscle or white adipose tissue, suggesting that this pathway might regulate hepatic metabolism.
Stimulation of hepatocytes with IL-4 shifted the metabolic reliance of these cells from fatty acid to glucose oxidation. STAT6, downstream of IL-4, was shown to directly interact with peroxisome proliferator-activated receptor- $\alpha$ (PPAR $\alpha)$ - an important regulator of the breakdown and oxidation of fatty acids - thereby inhibiting its transcriptional activity. This suggests that IL-4-STAT6 signalling inhibits fatty acid breakdown in hepatocytes by inhibiting PPARa.

The authors next determined whether IL-4-STAT6 signalling affected metabolism during obesity. Although Stat6 ${ }^{-1-}$ mice were resistant to high-fat diet-induced obesity owing to increased energy

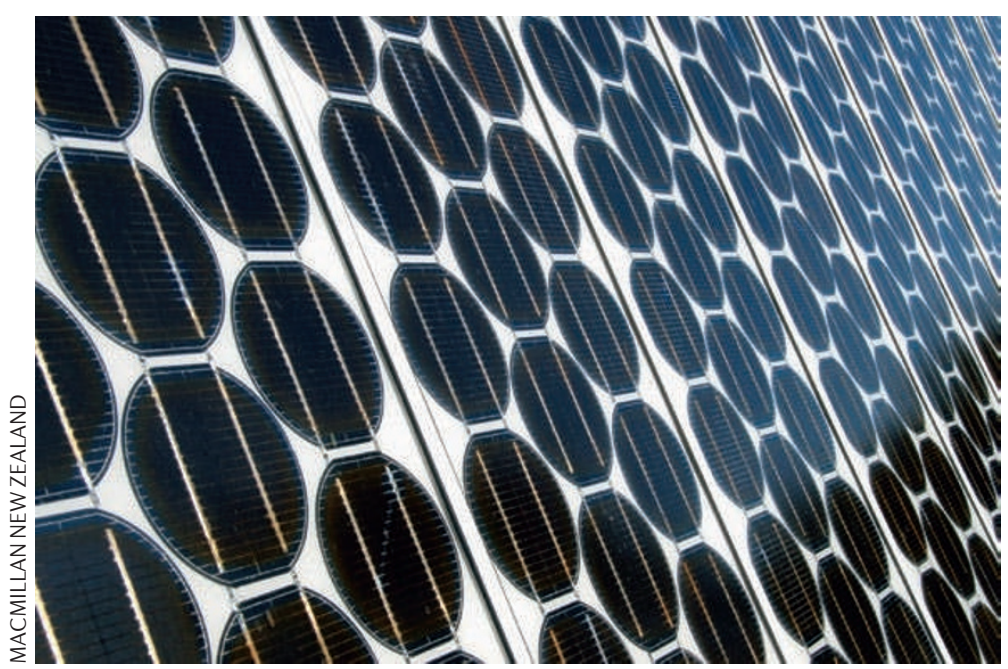

expenditure, they exhibited decreased insulin sensitivity. This decrease in insulin action, which was primarily observed in the liver, resulted from a loss of STAT6-mediated inhibition of PPARa, a transcription factor that has previously been shown to antagonize the anabolic actions of insulin and decrease glucose disposal.

Conversely, administration of IL-4 to wild-type mice on a high-fat diet improved glucose tolerance and insulin sensitivity. In this case, IL-4 treatment inhibited PPARa transcriptional activity in the liver, and attenuated the expression of nuclear factor- $\kappa \mathrm{B}(\mathrm{NF}-\kappa \mathrm{B})$-regulated inflammatory genes in white adipose tissue. Finally, using a model of allergic inflammation, the authors showed that polarization towards a $\mathrm{T}_{\mathrm{H}} 2$-type immune response in high-fat diet-fed mice improves glucose tolerance and insulin sensitivity.

Thus, this report highlights that IL-4-STAT6 signalling, which is associated with $\mathrm{T}_{\mathrm{H}}$ 2-type immune responses, improves insulin sensitivity and may protect against the disease-promoting effects of high-fat feeding.

Olive Leavy

ORIGINAL RESEARCH PAPER

Ricardo-Gonzalez, R. R. et al. IL-4/STAT6 immune axis regulates peripheral nutrient metabolism and insulin sensitivity. Proc. Natl Acad. Sci. USA 107, 22617-22622 (2010) 